\title{
Errors in the Use of NMR to Test Molecular Mobility during a Chemical Reaction
}

Lucy Fillbrook, Jan-Philipp Günther, Günter Majer, William S. Price, Peer Fischer, Jonathon Beves

Submitted date: 25/03/2021 - Posted date: 29/03/2021

Licence: CC BY-NC-ND 4.0

Citation information: Fillbrook, Lucy; Günther, Jan-Philipp; Majer, Günter; Price, William S.; Fischer, Peer; Beves, Jonathon (2021): Errors in the Use of NMR to Test Molecular Mobility during a Chemical Reaction. ChemRxiv. Preprint. https://doi.org/10.26434/chemrxiv.14306771.v1

A study reported in The Journal of Physical Chemistry Letters (12 (2021) 2370) of "boosted mobility" measured by diffusion NMR experiments contains significant errors in data analysis and interpretation. We carefully reanalyzed the same data and find no evidence of boosted mobility, and we identify several sources of error.

File list (2)

boosted mobility preprint JEB.pdf (540.59 KiB) view on ChemRxiv - download file 


\title{
Errors in the Use of NMR to Test Molecular Mobility during a Chemi- cal Reaction
}

\author{
Lucy L. Fillbrook, ${ }^{\ddagger, 1}$ Jan-Philipp Günther, ${ }^{\ddagger 2,3}$ Günter Majer, ${ }^{2}$ William S. Price, ${ }^{4}$ Peer Fischer, ${ }^{*}, 2,3$ and \\ Jonathon E. Beves., ${ }^{*}$ \\ ${ }^{1}$ School of Chemistry, UNSW Sydney, Sydney, NSW 2052, Australia. \\ ${ }^{2}$ Max Planck Institute for Intelligent Systems, Heisenbergstr. 3, 70569 Stuttgart, Germany. \\ ${ }^{3}$ Institute of Physical Chemistry, University of Stuttgart, Pfaffenwaldring 55, 70569 Stuttgart, Germany. \\ ${ }^{4}$ Nanoscale Group, School of Science, Western Sydney University, Penrith, NSW 2751, Australia.
}

KEYWORDS enhanced diffusion, nuclear magnetic resonance, diffusion NMR, spin relaxation

\begin{abstract}
A study reported in The Journal of Physical Chemistry Letters (12 (2021) 2370) of "boosted mobility" measured by diffusion NMR experiments contains significant errors in data analysis and interpretation. We carefully reanalyzed the same data and find no evidence of boosted mobility, and we identify several sources of error.
\end{abstract}

Claims of "boosted mobility" during chemical reactions have been based on nuclear magnetic resonance (NMR) diffusion measurements. A recent study ${ }^{1}$ purports to clarify issues related to these measurements, but the analysis is inconsistent with the reported data. Here we reanalyze the data provided in Ref. 1 and show that there is no evidence of boosted mobility (or enhanced diffusion). Since the conclusions of Ref. 1 have potentially far-reaching consequences, it is important that readers are alerted to these inconsistencies. This most recent publication $^{1}$ builds on previous claims ${ }^{2}$ which do not properly account ${ }^{3}$ for known problems in NMR diffusion measurements that have been highlighted in Ref. 4 .

The NMR diffusion data used in Ref. 1 is publicly accessible in data repositories ${ }^{5-7}$ and can be processed to obtain selfdiffusion coefficients for chemical species by fitting the Stejskal-Tanner equation: ${ }^{8}$

$$
I=I_{0} \cdot \exp \left[-D \gamma^{2} g^{2} \delta^{2}\left(\Delta-\frac{\delta}{3}\right)\right]
$$

where $g$ is the amplitude of the applied magnetic field gradient pulse of duration $\delta, \gamma$ is the gyromagnetic ratio, $\Delta$ is the diffusion time, and $I_{0}$ is the intensity that would be observed in the absence of the gradient pulse. Eq. (1) pertains to a single diffusing species and $I_{0}$ implicitly contains terms for signal attenuation due to spin-relaxation that occurs during the sequence.

Herein we critically assess the data reported in Ref. 1, using established methods, ${ }^{9-10}$ for a copper(I)-catalyzed azidealkyne cycloaddition reaction (Scheme 1). We highlight important problems with the reported data analysis in Ref. 1, namely: i) the arbitrary normalization of the diffusion coefficients of molecular species which leads to subsequent (incorrect) claims of "boosted mobility"; ii) overlapping NMR signals result in biased diffusion values towards the end of the reaction; iii) the inconsistently changing integral intensities over time, likely due to time-dependent spin-lattice relaxation rates, which prevent the measurement of accurate kinetic data; iv) the effect of concentration changes during individual diffusion measurements; and v) several incorrect claims concerning the design and operation of NMR probes and ancillary equipment for generating magnetic gradient pulses.

Scheme 1. The copper(I) catalyzed azide-alkyne "click" reaction between azide 1 and alkyne 2. $^{a}$

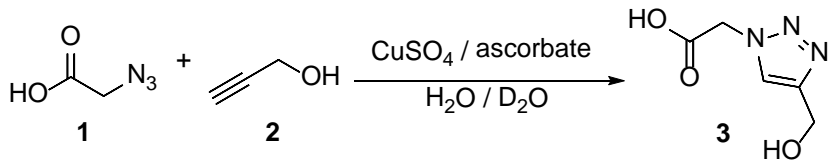

\footnotetext{
a Reaction conditions: ${ }^{1} 250 \mathrm{mM}$ of each reactant, $80 \mathrm{mM}$ sodium ascorbate, $20 \mathrm{mM} \mathrm{CuSO}_{4}$ catalyst.
}

\section{i) Arbitrary normalization of diffusion coefficients can give the appearance of "boosted mobility"}

Diffusion NMR data have been provided ${ }^{12}$ for a solution of azide 1, alkyne 2 and sodium ascorbate in $\mathrm{D}_{2} \mathrm{O}$ : that is, identical to Scheme 1 but excluding the (pre)catalyst copper sulfate. From this data, translational diffusion coefficients, $D$, were calculated for each species in this static (control) solution (Table 1). These diffusion coefficients, independently verified in our laboratories, ${ }^{11}$ will be referred to as $D_{0}$ values and are suitable for comparison to the time-resolved $D$ values obtained during the click reaction. ${ }^{13-15}$ Curiously, rather than compare these $D_{0}$ values from the control experiment with the diffusion coefficients measured during the reaction, the time-resolved $D$ values in Refs. $1-3$ were normalized to arbitrary " $D_{0}$ " values from an imprecisely defined "end" of the reaction (Table 1 , last four columns). Hereafter, these " $D_{0}$ " values "at the end of the reaction" are denoted as $D_{\infty}$ to reflect what they nominally represent. For species consumed during the reaction-azide 1 and alkyne $\mathbf{2}$ - the $D_{\infty}$ values are, by definition, for species with zero concentration, and therefore are, at best, determined 
Table 1. Diffusion coefficients of starting materials in a static solution and during the click reaction shown in Scheme 1. ${ }^{\mathrm{a}}$

\begin{tabular}{|c|c|c|c|c|c|c|}
\hline \multirow{3}{*}{$\begin{array}{l}\text { Chemical } \\
\text { species }\end{array}$} & \multirow{3}{*}{$\begin{array}{c}\text { Chemical } \\
\text { shift } \\
(\mathrm{ppm})^{\mathrm{b}}\end{array}$} & \multicolumn{5}{|c|}{ Diffusion coefficients $\left(\times 10^{-10} \mathrm{~m}^{2} \mathrm{~s}^{-1}\right)$} \\
\hline & & \multirow{2}{*}{$\begin{array}{c}D_{0} \text { from } \\
\text { static } \\
\text { control } \\
\text { sample }^{\mathrm{C}}\end{array}$} & \multirow{2}{*}{$\begin{array}{l}D_{\infty} \text { from } \\
\text { from "end" } \\
\text { of reaction } \\
(\text { Ref. } 2)^{\mathrm{d}}\end{array}$} & \multicolumn{3}{|c|}{$\begin{array}{c}D_{\infty} \text { from "end" } \\
\text { of reaction }\end{array}$} \\
\hline & & & & Inc. $^{f}$ & Dec. & Ran. $^{\mathrm{h}}$ \\
\hline HDO & 4.8 & 18.3 & 19.26 & $18.2^{\mathrm{i}}$ & $16.8^{\mathrm{j}}$ & $17.5^{\mathrm{k}}$ \\
\hline Azide & 4.0 & 7.7 & 6.08 & $6.5^{\mathrm{i}}$ & $6.0^{\mathrm{j}}$ & $6.4^{\mathrm{k}}$ \\
\hline Alkyne & 4.1 & 10.4 & 5.23 & $5.9^{\mathrm{i}}$ & $6.5^{\mathrm{j}}$ & $5.8^{\mathrm{k}}$ \\
\hline Alkyne & 2.7 & 10.4 & 5.43 & $5.2^{1}$ & $5.5^{\mathrm{m}}$ & $5.9^{\mathrm{n}}$ \\
\hline Product & 5.2 & - & n.r. ${ }^{\mathrm{q}}$ & $4.9^{\mathrm{i}}$ & $4.8^{\mathrm{j}}$ & $4.9^{\mathrm{k}}$ \\
\hline Ascorbate & 3.7 & 5.3 & 4.79 & $5.3^{\mathrm{i}}$ & $5.1^{\mathrm{j}}$ & $5.1^{\mathrm{k}}$ \\
\hline $\begin{array}{c}\text { Dehydroas- } \\
\text { corbic } \\
\text { acid }^{\circ}\end{array}$ & 4.6 & - & $4.67^{\mathrm{p}}$ & $5.1^{\mathrm{i}}$ & $5.3^{\mathrm{j}}$ & $4.8^{\mathrm{k}}$ \\
\hline
\end{tabular}

a Data available online. ${ }^{5-7}$ b Chemical shifts vary during the reaction, so are given here to one decimal place. ${ }^{\mathrm{C}}$ Measured in a sample of azide $\mathbf{1}$, alkyne 2 , sodium ascorbate. ${ }^{12}$ d Values taken from Tables S3, S4 in Ref 2. ${ }^{\mathrm{e}}$ Times selected as the final data point provided or, for the alkyne peak at $2.7 \mathrm{ppm}$, when no peak was visible. Values in italic highly depend on the integration regions (see Section ii)). ${ }^{\mathrm{f}}$ Inc. = monotonically increasing gradient amplitudes; ${ }^{13}$ g Dec. $=$ monotonically decreasing gradient amplitudes $;{ }^{14}$ ${ }^{\mathrm{h}}$ Ran. = random ("shuffled”) gradient amplitudes. ${ }^{15} \mathrm{i} t=100 \mathrm{~min} .{ }^{\mathrm{j}}$ $\mathrm{t}=121 \mathrm{~min}^{\mathrm{k}}{ }_{\mathrm{t}}=107 \mathrm{~min},{ }^{\mathrm{l}} \mathrm{t}=79 \mathrm{~min},{ }^{\mathrm{m}} \mathrm{t}=96 \mathrm{~min},{ }^{\mathrm{n}} \mathrm{t}=59 \mathrm{~min}$, ${ }^{\mathrm{o}}$ Chemical shift in agreement with literature, ${ }^{16} \mathrm{p}$ Assigned as "catalyst ligand in [...] chelating form" at 4.4 ppm in Ref. $2 .{ }^{9}$ n.r. $=$ not reported.

at times when the concentrations of these species are very low rather than when the reaction is complete. The arbitrary time points selected to measure $D_{\infty}$ for different species varies between experiments.

We reanalyzed the diffusion data for the three reactant signals discussed above, obtained with either monotonically increasing $(\boldsymbol{\Delta}),^{13}$ monotonically decreasing $(\boldsymbol{\nabla})^{14}$ or random $(\bullet)^{15}$ gradient pulse amplitudes, and compared the resulting $D$ values with the $D_{0}$ values from the static control sample (Figure 1). Neither of the $D$ values derived from either alkyne signal (Figure 1a,b) exhibited any enhanced diffusion as claimed in Figure 2E of Ref. 1. In fact, this species appears to be diffusing more slowly at all times during the reaction than in the static control sample. The diffusion decrease has been misrepresented as "boosted mobility" in Ref. 1 due to an erroneous normalization of $D$ to arbitrarily chosen $D_{\infty}$ values.

The representation of the diffusion coefficients in Figure 1 as absolute values also negates the claim ${ }^{1}$ that "the ratio of the apparent diffusion coefficient obtained by a random sequence measurement, to that from linearly (sic) increasing sequence measurement, is unity within experimental uncertainty at all reaction times", which is clearly not true for the azide signal (4.1 ppm; Figure 1c). The dependence of the diffusion coefficient on the order of gradients applied implies that the "quasisteady-state condition" assumed by the authors of Ref. 1 does not hold.

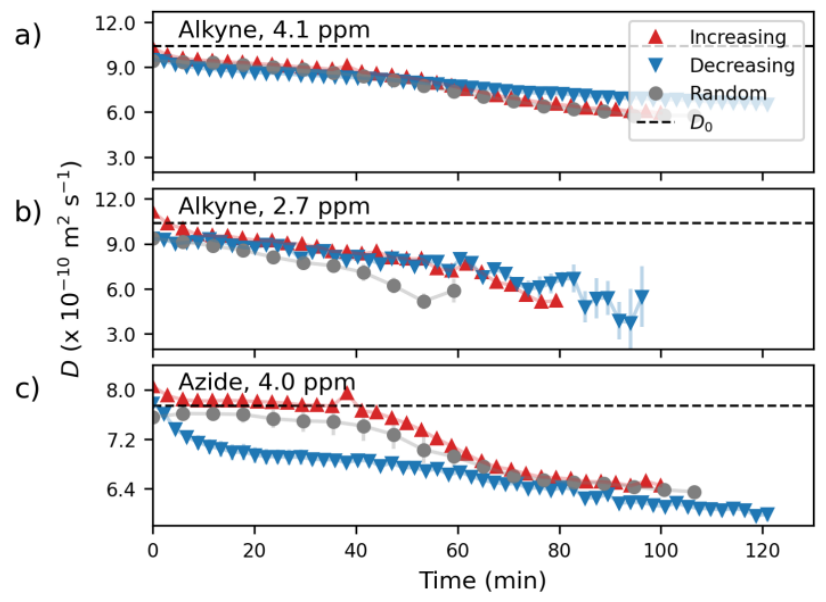

Figure 1. No "boosted mobility". Comparison of time-resolved diffusion coefficients $(D)$ calculated using monotonically increasing $(\boldsymbol{\Delta}),{ }^{13}$ monotonically decreasing $(\boldsymbol{\nabla})^{14}$ and random $(\bullet)^{15}$ gradient pulse amplitude lists, data from Ref. 1 . The $D_{0}$ is the $D$ obtained in a static control NMR diffusion experiment on a sample identical to the reaction solution, excluding the catalyst. ${ }^{12}$ The vertical scale in c) differs from that in a) and b).

A clearer demonstration of the effect of arbitrary selection of $D_{\infty}$ is shown in Figure 2. The absolute measured diffusion coefficients using monotonically increasing, ${ }^{13}$ monotonically decreasing $^{14}$ or random ${ }^{15}$ gradient pulse amplitudes, for the alkyne signal $(4.1 \mathrm{ppm})$ is shown in Figure 2a. The $D_{0}$ value for this species measured in the control experiment is shown as a black dotted line $\left(D_{0}=10.4 \times 10^{-10} \mathrm{~m}^{2} \cdot \mathrm{s}^{-1}\right)$. The $D_{\infty}$ values, taken as the $D$ at the end of each experiment, are indicated with dotted lines at the bottom of Figure 2a, highlighting how a different $D_{\infty}$ is used in each case.

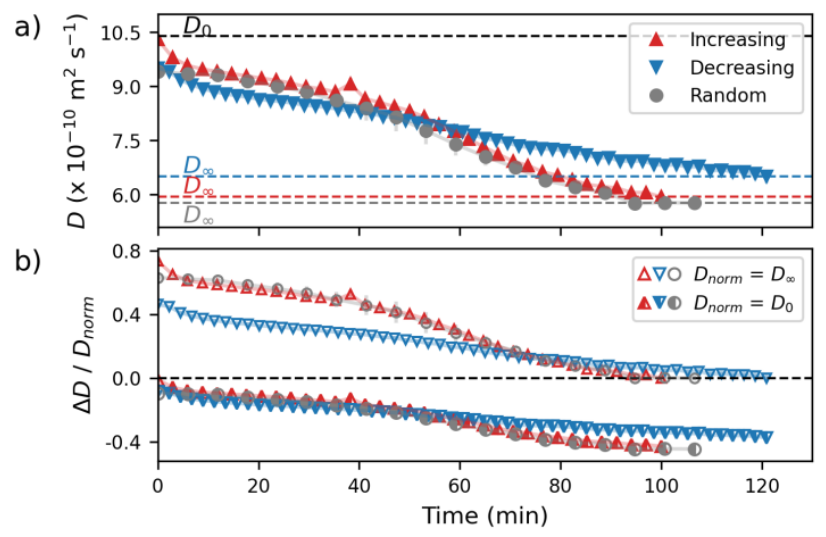

Figure 2. Arbitrary definitions of $D_{\infty}$ lead to differences in perceived "boosted mobility". Data from Ref. 1 is shown for the alkyne signal at $4.1 \mathrm{ppm}$ during the reaction with monotonically increasing $(\boldsymbol{\Delta}),{ }^{13}$ monotonically decreasing $(\boldsymbol{\nabla})^{14}$ or random $(\bullet)^{15}$ gradient amplitude ordering.

The apparent change in diffusion $\left(\Delta D / D_{0}\right.$ or $\left.\Delta D / D_{\infty}\right)$ using the $D_{0}$ and $D_{\infty}$ values (Figure 2a) are shown in Figure 2b. It is evident that when $D_{0}$ values are used the diffusion is apparently slower during the reaction in all cases. In contrast, when the 
arbitrary $D_{\infty}$ values are used, an apparent (i.e. artefactual) increase in diffusion ("boosted mobility") appears.

While the authors of Ref. 1 should have referenced their measurements to the correct $D_{0}$ values, it is apparent that this alone does not account for all the artefacts in Ref. 1 as other effects must be causing the gradual decrease in apparent diffusion coefficients during the reaction. ${ }^{17}$

\section{ii) Overlapping NMR peaks lead to incorrect diffusion measurements}

Measuring diffusion coefficients of overlapping signals is fraught with difficulty where the contributing signals come from species with similar diffusion coefficients. ${ }^{18}$ We find that, despite claims of "cleanly resolved" peaks, there is significant NMR signal overlap for peaks of interest. The overlap between the key signals of the azide and alkyne starting materials with signals from the sodium ascorbate (or products formed from the ascorbate oxidation, e.g. dehydroascorbic acid) is clearly evident in Figure 3 (and in the inset of Figures 3A-B in Ref. 1, here Figure 3a).

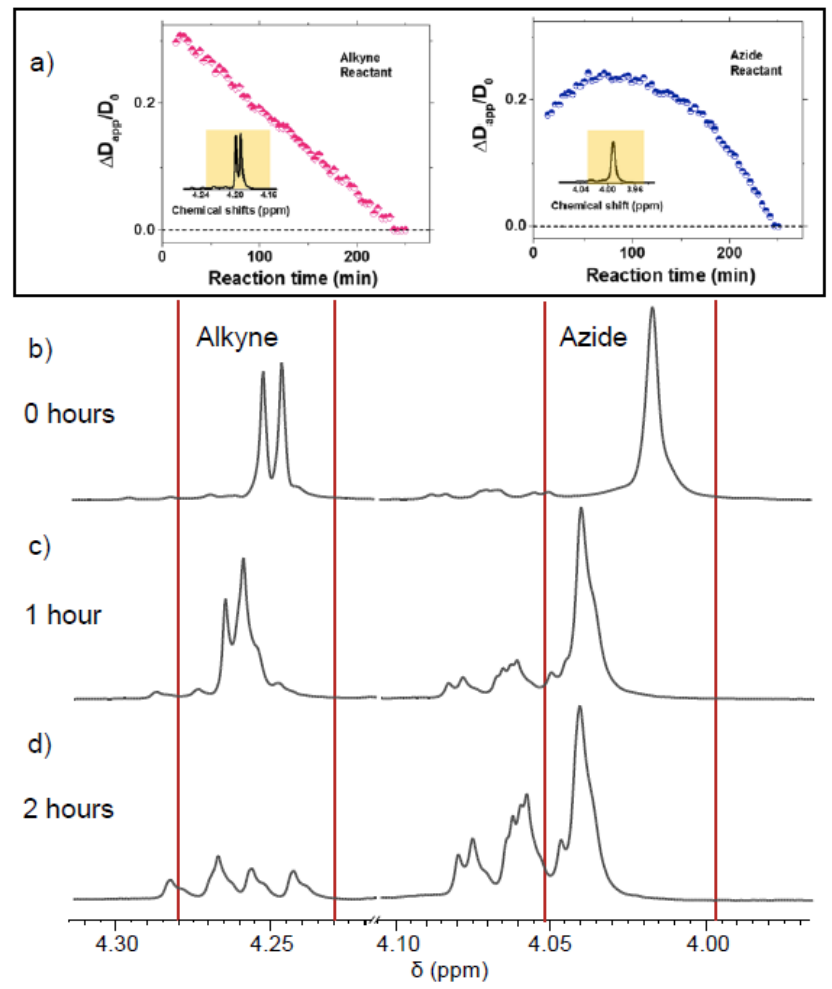

Figure 3. a) The claimed "cleanly resolved" ${ }^{1} \mathrm{H}$ NMR signals of the alkyne and azide reactants as shown in Ref. 1. b) The same signals at various stages during the reaction (using the lowest gradient amplitude), ${ }^{13}$ in each case normalized to the maximum peak height for ease of comparison. Towards the end of the reaction (when $D_{\infty}$ is selected in Ref. 1 ) the signals are dominated by the sodium ascorbate/dehydroascorbate peaks at slightly higher chemical shift. Vertical red lines indicate approximate regions of integration. a) Reprinted with permission from J. Phys. Chem. Lett. 2021, 12, 2370-2375. Copyright 2021 American Chemical Society.

As the reactants are consumed during the reaction, the fraction of the non-reactant signals in the integrated area increases, until only the signals from the non-reactant compound(s) re- main (if the reaction reaches completion). These non-reactant compounds have lower diffusion coefficients than the reactants with both dehydroascorbic acid and sodium ascorbate having similar diffusion coefficients at the end of the reaction $\left(D_{\infty} \approx 5 \times 10^{-10} \mathrm{~m}^{2} \cdot \mathrm{s}^{-1}\right.$, Table 1$)$. The static control sample provides the most reliable comparison, where sodium ascorbate $\left(D_{0}=5.3 \times 10^{-10} \mathrm{~m}^{2} \cdot \mathrm{s}^{-1}\right)$ diffuses at roughly half the rate of the alkyne $\left(D_{0}=10.4 \times 10^{-10} \mathrm{~m}^{2} \cdot \mathrm{s}^{-1}\right)$ and $30 \%$ slower than the azide $\left(D_{0}=7.7 \times 10^{-10} \mathrm{~m}^{2} \cdot \mathrm{s}^{-1}\right)$. Therefore, the $D_{\infty}$ used for normalization in Ref. 1 is dominated by a slower diffusing species, which is probably the major cause for the observed apparent diffusion decrease during the reaction. The reactant signals cannot be separated from those of the sodium ascorbate/dehydroascorbic acid due to extensively overlapped peaks. Despite deceivingly good data fitting (with Eq. 1), overlapping peaks hinder reliable diffusion analysis and are a major contributor to the incorrect conclusions of Refs. 1-3.

\section{iii) Invalid kinetic data}

The relative NMR signal integrals for the azide reactant (1), product (3) and solvent (residual HDO) during the reaction as presented in Figure 2B of Ref. 1 are reproduced below as Figure 4a. We have reanalyzed their data ${ }^{13}$ and present the result in Figure 4b. The integral of the residual solvent signal is not constant during the reaction. As the concentration of the solvent cannot be changing over time in this reaction, the change in the residual solvent signal clearly indicates that other factors must cause the change in the signal intensity. The $>50 \%$ intensity loss of the integrated solvent signal has no chemical explanation and is seemingly an artefact caused by spin relaxation changes, consistent with our previous observations. ${ }^{4}$
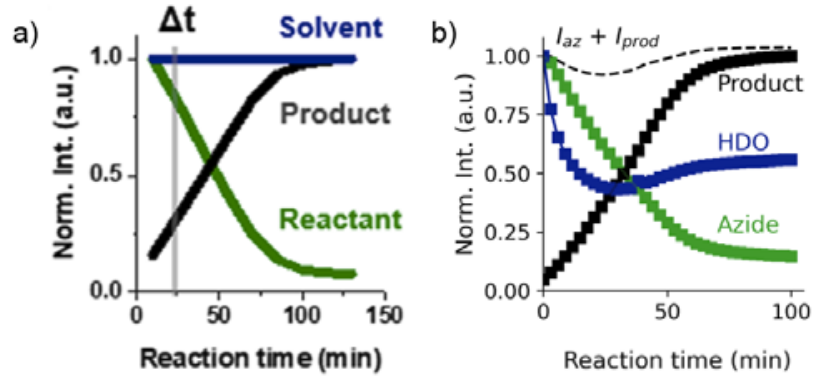

Figure 4. NMR peak integration over time. a) "Smoothed" data reported in Figure 1 of Ref. 1, claiming a constant integral for the residual solvent (HDO) signal. b) Signal intensities $\left(I_{0}\right)$ during the reaction for the azide signal $\left(I_{\mathrm{az}}\right)$ and the product peak at $5.2 \mathrm{ppm}$ $\left(I_{\text {prod }}\right)$. The raw diffusion data ${ }^{13}$ (consisting of 7 gradient values) was analyzed using Eq. (1) to provide estimates of both $D$ and $I_{0}$ values at each time point. Each $I_{0}$ value was normalized by the maximum value for each peak during the whole reaction. a) Reprinted with permission from J. Phys. Chem. Lett. 2021, 12, 23702375. Copyright 2021 American Chemical Society.

Ignoring signal losses due to relaxation during the diffusion NMR sequence, the sum of the integrals of the starting material (azide, $I_{\mathrm{az}}, 4.0 \mathrm{ppm}$ ) and product ( $I_{\text {prod }}, 5.2 \mathrm{ppm}$ ) should be constant, but this is not the case (Figure $4 \mathrm{~b}$, dashed line). As the signal integrations are no longer proportional to concentrations, it then follows that any reaction rates concluded from these integrals will be incorrect. 


\section{iv) The effect of concentration (or relaxation rate) changes during measurements}

It is known that if changes in concentration are correlated with the order of applied gradient amplitudes used during NMR diffusion measurements then erroneous diffusion coefficients will result. ${ }^{19,20,4}$ Using the reaction rate in Ref. 1 (40 $\mu \mathrm{M} / \mathrm{s}$, which might be incorrect, see Section iii)) and considering the shortest measurement time used (3 min), we find signal changes for both azide 1 and alkyne 2 of up to 11\% over a single diffusion measurement. This contradicts the claim that these changes are "at most $3 \%$ " which is only true for the first 9 min of the reaction. While this intensity change due to concentration is not the dominant cause of the erroneous results in Ref. 1, the spin-relaxation induced intensity changes will have an even more significant impact-as was the case for the residual HDO signal that was the focus of the original claims. ${ }^{2}$

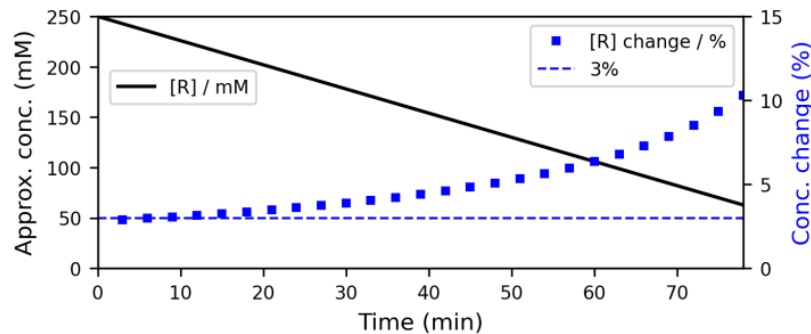

Figure 5. The changes in the concentration of the alkyne and azide reactants over the first 80 minutes of the reaction. Figure 2C of Ref. 1 claims a "constant reaction rate for the first $80 \mathrm{~min}$ ", given as $40 \mu \mathrm{M} / \mathrm{s}$ in. During one diffusion measurement (3 min) the percentage change in signal intensity due to concentration change exceeds 3\% (dotted line) for all times after 9 mins from the start of the reaction.

The use of shuffled gradient lists to remove the correlation between changes in concentration (or relaxation) with the ordering of applied gradients allows more accurate determination of diffusion coefficients in these circumstances. ${ }^{19-21,4}$ While the authors of Ref. 1 claim to use shuffled gradient amplitudes, it is apparent that the gradients used in Ref. 1 are still correlated, as can be seen in Figure 6. The positive correlation of the random order used in Ref. 1 contributes to the similarity in the observed diffusion coefficients obtained from increasing and random gradient order in Figure 1.

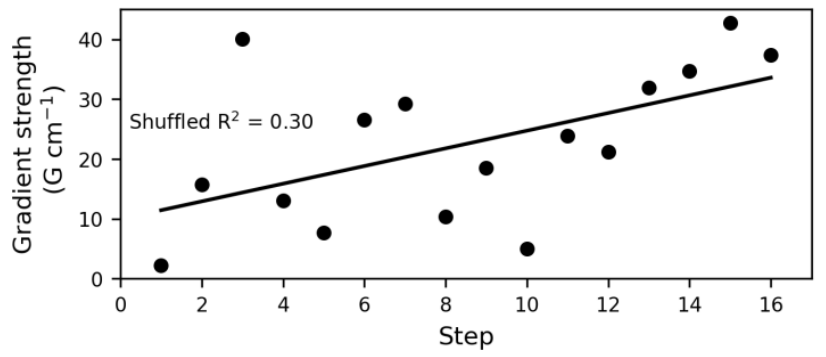

Figure 6. The order of gradient amplitudes used in Ref. 1 maintains a positive correlation. A more optimally shuffled list would have an $\mathrm{R}^{2}$ close to zero, e.g. 0.01 .

\section{v) Spectrometer performance and other technical concerns}

In Ref. 1 it is stated that "it is reasonable that they [eddy currents] may differ according to the sequence of magnetic field gradients (increasing, decreasing, and random)". The gradient generation systems in contemporary NMR spectrometers are capable of producing highly reproducible gradient pulses with eddy currents not normally becoming significant until gradient amplitudes exceed several hundred $\mathrm{G} \cdot \mathrm{cm}^{-1} \cdot{ }^{21,10}$ The generation of eddy currents is even less likely when sineshaped gradient pulses ${ }^{23}$ are used since the severity of eddy currents is directly related to the rate of the rise and fall of the gradient pulses. Even then, the eddy currents generated on a typical high-resolution probe containing shielded gradient coils after modest gradient amplitude pulses (i.e., $~ 50 \mathrm{G} \cdot \mathrm{cm}^{-1}$ ) would dissipate in under $1 \mathrm{~ms}$. Thus, there can be no correlation between eddy currents and whether diffusion measurements are performed with random or monotonically increasing or decreasing gradients.

The authors of Ref. 1 claim that NMR spectrometers are not designed for shuffled gradients: "we speculate that hardware may influence this, as the NMR instrument was designed and calibrated for linear sequences, whereas we randomized the sequence using a nonuniform sampling method." Accurate gradient calibration is not related to the order of the gradient amplitudes.

There is seemingly confusion in Ref. 1 as to the mechanism of NMR diffusion measurements. The claim that "the only signal left" after a diffusion NMR experiment "is from chemical moieties that migrated to a different vertical location in the field gradient" is incorrect. Migration in the direction of the applied field gradient leads to greater signal attenuation. ${ }^{10,24}$ Further, they used a linear fit to linearized NMR diffusion data (i.e., $\log (I)$ vs. $g^{2}$ plot), which leads to incorrect weighting of error amplitudes. ${ }^{10}$ Instead non-linear regression of the Stejskal-Tanner equation to the experimental data should have been used. ${ }^{10}$

\section{Conclusion}

When monitoring the diffusion of a molecular species during a reaction, the measured diffusion coefficient must be compared to a reasonable reference value. The diffusion coefficients of all species in the absence of the catalyst should be used to normalize time-resolved diffusion coefficients. However, in Refs. 1-3 the diffusion coefficients are normalized to the apparent diffusion coefficients at arbitrary, and inconsistent, time points towards the end of the reaction. This selective choice of normalization biases the analysis for the different molecular species and underlies the erroneous conclusions in Refs. 1-3, We show that the supposedly "cleanly resolved" ${ }^{1} \mathrm{H}$ NMR signals are overlapped with signals for other species, and that this overlap changes during the course of the reaction. This strongly influences the calculated diffusion coefficients and calls into question the peak integration and kinetic analysis presented in Ref. 1 and is a major contributor to the incorrect claims of "boosted molecular mobility" in Refs. 1-3. 


\section{ASSOCIATED CONTENT}

Supporting Information. Processed NMR data is provided. Python codes for processing data are publically available at https://github.com/tscmacdonald/diffusion-fits/pull/1.

\section{AUTHOR INFORMATION}

\section{Corresponding Author}

* Jonathon E. Beves - School of Chemistry, UNSW Sydney, NSW 2052, Australia; ORCID: 0000-0002-5997-6580; Email: j.beves@unsw.edu.au.

* Peer Fischer - Max Planck Institute for Intelligent Systems, Heisenbergstr. 3, 70569 Stuttgart, Germany; Institute of Physical Chemistry, University of Stuttgart, Pfaffenwaldring 55, 70569 Stuttgart, Germany; ORCID: 0000-0002-8600-5958; Email: fischer@is.mpg.de.

\section{Authors}

Lucy L. Fillbrook - School of Chemistry, UNSW Sydney, NSW 2052, Australia; ORCID: 0000-0002-2219-6759

Jan-Philipp Günther - Max Planck Institute for Intelligent Systems, Heisenbergstr. 3, 70569 Stuttgart, Germany; Institute of Physical Chemistry, University of Stuttgart, Pfaffenwaldring 55, 70569 Stuttgart, Germany. ORCID: 0000-00028083-0658

Günter Majer - Max Planck Institute for Intelligent Systems, Heisenbergstr. 3, 70569 Stuttgart, Germany.

William S. Price - School of Science, Western Sydney University, Penrith, NSW 2751, Australia, ORCID: 0000-00028549-4665

\section{Author Contributions}

The manuscript was written through contributions of all authors. All authors have given approval to the final version of the manuscript. $\ddagger$ These authors contributed equally.

\section{Funding Sources}

This work was supported by the Australian Research Council (FT170100094 to J.E.B.) and UNSW Australia (L.L.F.).

\section{Notes}

The authors declare no competing financial interests.

\section{ACKNOWLEDGMENT}

We thank Dr Klaus Zick from Bruker Biospin, and Dr Thomas MacDonald at UNSW School of Physics for fruitful discussions. We acknowledge the Mark Wainwright Analytical Centre at UNSW Sydney for access to the NMR facility.

\section{REFERENCES}

(1) Wang, H.; Huang, T.; Granick, S. Using NMR to Test Molecular Mobility during a Chemical Reaction. J. Phys. Chem. Lett. 2021, 12, 2370-2375.

(2) Wang, H.; Park, M.; Dong, R.; Kim, J.; Cho, Y.-K.; Tlusty, T.; Granick, S. Boosted molecular mobility during common chemical reactions. Science 2020, 369, 537-541.

(3) Wang, H.; Park, M.; Dong, R.; Kim, J.; Cho, Y.-K.; Tlusty, T.; Granick, S. Response to Comment on "Boosted molecular mobility during common chemical reactions”. Science 2021, 371, eabe8678.

(4) Günther, J.-P.; Fillbrook, L. L.; MacDonald, T. S. C.; Majer, G.; Price, W. S.; Fischer, P.; Beves, J. E. Comment on "Boosted molecular mobility during common chemical reactions”. Science 2021, 371, eabe8322.
(5) Wang, H.; Huang, T.; Granick, S., Use or misuse of NMR to test molecular mobility during chemical reactions (updated) all 2021; https://zenodo.org/record/4515126.

(6) Wang, H.; Huang, T.; Granick, S., Use or misuse of NMR to test molecular mobility during chemical reactions 2021; https://zenodo.org/record/4472031.

(7) Wang, H.; Park, M.; Dong, R.; Kim, J.; Cho, Y.-K.; Tlusty, T.; Granick, S., Raw data for Wang et al., "Boosted molecular mobility during common chemical reactions," Science (2020) 369, 537-541. 2021; https://zenodo.org/record/4553903.

(8) Stejskal, E. O.; Tanner, J. E. Spin Diffusion Measurements: Spin Echoes in the Presence of a Time-Dependent Field Gradient. J. Chem. Phys. 1965, 42, 288-292.

(9) Price, W. S. Pulsed-field gradient nuclear magnetic resonance as a tool for studying translational diffusion: Part II. Experimental aspects. Concepts Magn. Reson. 1998, 10, 197-237.

(10) Price, W. S. NMR Studies of Translational Motion: Principles and Applications; Cambridge University Press: Cambridge, 2009, DOI: 10.1017/CBO9780511770487.

(11) Measured for a solution of $250 \mathrm{mM}$ azide 1, $250 \mathrm{mM}$ alkyne 2 in $\mathrm{D}_{2} \mathrm{O}, 500 \mathrm{MHz}, \delta=2 \mathrm{~ms}, \Delta=50 \mathrm{~ms}, 16$ gradient ampltiudes from 2.448 G.cm-1. A separate sample of sodium ascorbate ( $80 \mathrm{mM}$ in $\left.\mathrm{D}_{2} \mathrm{O}\right)$ was measured under the same NMR diffusion conditions.

(12) Wang, H.; Park, M.; Dong, R.; Kim, J.; Cho, Y.-K.; Tlusty, T.; Granick, S., Raw data for Wang et al., "Boosted molecular mobility during common chemical reactions," Science (2020) 369, 537-541. Dataset: 20190104-click-control-no Cu. 2021; https://zenodo.org/record/4553903.

(13) Wang, H.; Huang, T.; Granick, S., Use or misuse of NMR to test molecular mobility during chemical reactions. Dataset: click reaction linearly-increasing gradients_400 MHz. 2021; https://zenodo.org/record/4515126.

(14) Wang, H.; Huang, T.; Granick, S., Use or misuse of NMR to test molecular mobility during chemical reactions. Dataset: click reaction linearly-decreasing gradients_400 MHz. 2021; https://zenodo.org/record/4515126.

(15) Wang, H.; Huang, T.; Granick, S., Use or misuse of NMR to test molecular mobility during chemical reactions, Dataset: Fig. 3 - click reaction random gradients_Bruker/Brucker-Random by non-uniform sampling-400MHz-20201021-NUS-nowater-2scan-1100us-5.5min per data file; 2021; https://zenodo.org/record/4472031.

(16) Belapure, S. A.; Beamer, Z. G.; Bartmess, J. E.; Campagna, S. R. A biomimetic synthesis of (-)-ascorbyl phloroglucinol and studies toward the construction of ascorbyl-modified catechin natural products and analogues. Tetrahedron 2011, 67, 9265-9272.

(17) We note that viscosity changes during the reaction can only have a minor effect on the diffusion coefficients since the apparent diffusion coefficient of the solvent (residual HDO) changes minimally ( $<8 \%$, Table 1).

(18) Antalek, B. Using pulsed gradient spin echo NMR for chemical mixture analysis: How to obtain optimum results. Concepts Magn Reson. 2002, 14, 225-258.

(19) MacDonald, T. S. C.; Price, W. S.; Astumian, R. D.; Beves, J. E. Enhanced Diffusion of Molecular Catalysts is Due to Convection. Angew. Chem. Int. Ed. 2019, 58, 18864-18867.

(20) MacDonald, T. S. C.; Price, W. S.; Beves, J. E. Time-Resolved Diffusion NMR Measurements for Transient Processes. ChemPhysChem 2019, 20, 926-930.

(21) Urbańczyk, M.; Bernin, D.; Czuroń, A.; Kazimierczuk, K. Monitoring polydispersity by NMR diffusometry with tailored norm regularisation and moving-frame processing. Analyst 2016, 141, 17451752 .

(22) Price, W. S.; Hayamizu, K.; Ide, H.; Arata, Y. Strategies for diagnosing and alleviating artifactual attenuation associated with large gradient pulses in PGSE NMR diffusion measurements. J. Magn. Reson. 1999, 139, 205-212.

(23) Equation 1 must be slightly modified to account for such pulses, see Ref. 10 for details.

(24) Gupta, A.; Stait-Gardner, T.; Price, W. S. NMR imaging and diffusion. Adsorption 2021, 10.1007/s10450-021-00298-9. 
Other files

Processed data.zip (554.69 MiB)

view on ChemRxiv - download file 